\title{
Le répertoire de théâtre jeunesse : des esthétiques contagieuses
}

Theater for Young People: A Contagious Repertoire

\section{Marie Bernanoce}

\section{OpenEdition}

\section{Journals}

Édition électronique

URL : https://journals.openedition.org/recherchestravaux/761

DOI : 10.4000/recherchestravaux.761

ISSN : 1969-6434

\section{Éditeur}

UGA Éditions/Université Grenoble Alpes

\section{Édition imprimée}

Date de publication : 1 décembre 2015

Pagination : 23-38

ISBN : 978-2-84310-314-8

ISSN : 0151-1874

\section{Référence électronique}

Marie Bernanoce, "Le répertoire de théâtre jeunesse : des esthétiques contagieuses 》, Recherches \& Travaux [En ligne], 87 | 2015, mis en ligne le 01 janvier 2017, consulté le 29 octobre 2021. URL : http:// journals.openedition.org/recherchestravaux/761 ; DOI : https://doi.org/10.4000/recherchestravaux. 761 
Marie BERNANOCE

Univ. Grenoble Alpes

UR LITT\&ARTS (LITEXTRA, CINESTHEA)

\title{
Le répertoire de théâtre jeunesse : des esthétiques contagieuses
}

\author{
LA PRINCESSE \\ Qu'est-ce que l'art? \\ LES SQUELETTES
}

Dire d'un mot la mort avec la joie.

Olivier $\mathrm{PY}^{\mathrm{I}}$

La réflexion menée ici ${ }^{2}$ se penchera sur les relations qui se tissent entre esthétique et éthique dans le répertoire de théâtre jeunesse ce qui, selon la terminologie que j'adopte depuis plusieurs années, concernera avant tout le théâtre publié dans des collections spécialisées donc les écritures, dans une perspective dramaturgique.

Après avoir exploré en quoi ces écritures relèvent d'une forme de détour, ce qui permet de donner un cadre esthétique aux questions posées par l'adresse aux enfants et aux jeunes, nous verrons comment se dégagent de grandes formes esthétiques et comment celles-ci sont indissolublement liées à des engagements éthiques.

I. O. Py, La Jeune Fille, le Diable et le Moulin, Paris, L'École des loisirs, coll. "Théâtre», I995, p. 44.

2. Cet article s'appuie sur le document original de mon dossier d'habilitation à diriger les recherches, présenté sous la direction de J. Danan, professeur des universités, IET, Paris 3 Sorbonne Nouvelle : «La voix didascalique dans le répertoire de théâtre jeunesse : esthétique et éthique, questions posées à son enseignement». 


\section{La jeunesse comme détour}

\section{Un théâtre mineur}

L'écrivain de thêâtre a aujourd'hui énormément de droits et de libertés. L'écriture théâtrale contemporaine peut presque tout se permettre, jusqu'à risquer sa dissolution dans des textes-matériaux sans ossature dramaturgique d'une part, jusqu’à poursuivre les mirages d'une écriture classique érigée en modèle tutélaire, d'autre part. Cette extrême ouverture esthétique définit le genre moins en compréhension qu'en extension, et le dramatique semble bel et bien reposer avant tout sur la rencontre ouverte à grands battants de deux désirs de scène, celui de l'auteur, ou de l'éditeur, et celui du lecteur, même quand ces désirs sont plus ou moins directs et conscients.

L'analyste doit alors se situer dans cette béance active. Il faut que les outils précis de l'analyse soient suffisamment dynamiques pour rendre compte de ce dialogue permanent à trois, l'auteur, la scène et le lecteur, incarné dans un matériau littéraire que l'on ne peut plus opposer à la théâtralité et qui peut aussi être le ferment de son renouveau du fait de défis à la scène poussant à l'innovation. Il ne s'agit plus de déterminer, dans une perspective formaliste, les éléments intrinsèques inhérents au genre mais de prendre toute la matière imprimée d'une pièce, y compris l'objet livre, pour la rendre vivante et l'infléchir vers la scène, imaginaire ou réelle. Telle est aujourd'hui, loin des perspectives sémiologique et formaliste trop linéaires, l'approche générique que l'on peut adopter pour étudier le théâtre contemporain.

À cela, le théâtre publié à destination des jeunes ajoute une autre dimension, elle aussi systémique : l'adresse aux jeunes, plus ou moins voulue par l'auteur ${ }^{3}$ et/ou réalisée par l'éditeur, fonctionne en effet comme un mode de détour, au sens que Jean-Pierre Sarrazac donne à ce mot, pour dire le monde, mais aussi se dire et dire le théâtre :

L'esprit de routine et de substitution soit nous fait trop coller à la réalité, soit nous en coupe irrémédiablement, souvent les deux à la fois : nous sommes dans un rapport de coalescence avec une réalité que nous ne voyons plus; nous nous engluons dans le «déjà connu». L'esprit du détour, lui, nous ouvre le chemin d'une reconnaissance : nous nous éloignons pour mieux nous rapprocher. Le

3. Nombreux sont les cas de pièces initialement publiées en collection généraliste et republiées ensuite en collection jeunesse, hors de toute volonté initiale de l'auteur. Voir mon article «L'engagement dans des pièces jeunesse qui n'en étaient pas : positionnement moral, éthique, esthétique?», dans B. Benert, P. Clermont (dir), Contre l'innocence. Esthétique de l'engagement en littérature de jeunesse, Francfort-sur-le-Main, Peter Lang, 20II, p. 75-87. 
détour permet un retour saisissant - étrangéifiant - sur cette réalité dont nous voulions témoigner ${ }^{4}$.

En ce sens, le théâtre jeunesse s'avère vivifiant, souvent "saisissant», et peut contribuer à nous "désengluer ", pour reprendre la métaphore développée par Jean-Pierre Sarrazac. Et telle est effectivement ma sensation de grande lectrice de ce théâtre, ce que Jean-Claude Grumberg, dans son mot d'auteur de Vers un théâtre contagieux, a traduit par cette définition : "Écrire pour la jeunesse, c'est donner de l'air ${ }^{5}$. Ce regard aéré sur le monde relève bien, me semble-t-il, d'une "déformation qui informe ${ }^{6}$ ".

Dans le prolongement des réflexions de Deleuze et Guattari ${ }^{7}$, le théâtre jeunesse peut ainsi apparaître comme l'une des formes d'un théâtre mineur, déterritorialisant, posant d'intéressantes questions à la notion d'adresse, si importante pour le théâtre. Nombreux sont les auteurs témoignant de leur surprise à voir la réaction des enfants face à leurs textes, lus ou mis en scène. Si l'adresse aux enfants et aux jeunes, qu'elle soit le fait de l'éditeur, de l'auteur ou du metteur en scène, prend en compte ce que sera la réception, c'est dans l'énigme de sa nature. L'adresse est toujours plus ou moins une fiction. Et ce geste porte intrinsèquement une dimension éthique. Le théâtre à l'intention des jeunes ne peut pas courir le risque de les désespérer ${ }^{8}$, et donc il construit un adulte auteur, lecteur et spectateur que ne saisit pas le désespoir. Par ailleurs, les lignes de partage qualitatif ne sont plus à penser en termes de spécificités du théâtre jeunesse par rapport au théâtre généraliste, ou du théâtre jeunesse par rapport au théâtre jeune public. Dans tous les cas, les grandes œuvres seront celles qui développent un mode de détour puissant, fécond et déstabilisant.

Dire le monde du point de vue de l'enfance comporte alors deux formes fondamentales de détour, le dramatique et l'épique, liées entre elles et jouant les jeux complexes de l'adhésion dramatique et de la distance épique. Or, de ce fait, il n'y a pas de tabous dans ce répertoire ${ }^{9}$ la distance épique permet de

4. J.-P. Sarrazac (dir.), Études théâtrales, $\mathrm{n}^{\circ}$ 22, Poétique du drame moderne et contemporain. Lexique d'une recherche, 200I, p. 37-38.

5. M. Bernanoce, Vers un théatre contagieux, Répertoire critique du théatre contemporain pour la jeunesse, vol. II, Montreuil, Éditions Théâtrales, 20I2, p. 224.

6. J.-P. Sarrazac (dir.), ouvr. cité, p. 37.

7. G. Deleuze et F. Guattari, Kafka: pour une littérature mineure, Paris, Minuit, 1985.

8. C'est ainsi la position de J. Danan dans son article, "Y a-t-il une spécificité de l'écriture théâtrale pour le jeune public?», dans C. Connan-Pintado, G. Behoteguy (dir.), Littérature de jeunesse au présent. Genres littéraires en question(s), Bordeaux, Presses universitaires de Bordeaux, coll. "Études sur le livre de jeunesse», 2015, p. I29-I35.

9. Nous entendons ici le mot répertoire dans son sens de corpus des œuvres publiées à disposition des lecteurs et metteurs en scène. 
tout évoquer, précisément parce que l'important est dans le fonctionnement des modes de récit adoptés par le sujet épique, pour reprendre l'expression de Peter Szondi ${ }^{\mathrm{ro}}$, que Monique Martinez-Thomas appelle le didascale ${ }^{\mathrm{II}}$ et que j'ai théorisé pour ma part sous la dénomination de "voix didascalique ${ }^{\mathrm{i} 2}$ ».

Il s'agit à mes yeux de sortir d'une conception intentionnaliste et donneuse d'ordre en matière de régie, de mise en scène à venir. Le texte de théâtre est un texte en désir de scène, au sens que Deleuze donnait à l'expression de machine désirante, et ce désir va nécessairement prendre différentes formes esthétiques à la réception. En lui et par lui, on peut rêver une langue, une fiction et une scène. Cela conduit à revoir les notions d'indications scéniques (conception fonctionnelle) et de texte didascalique (conception formaliste) au profit de la notion de voix didascalique, de nature pragmatique : il s'agit de chercher ce qui peut apparaître, dans les fonctionnements du texte de théâtre, comme les lieux privilégiés de rencontre et de tension entre figure de l'auteur (Maurice Couturier ${ }^{\mathrm{r}}$ ), figure de la scène (modèle de représentation de Bernard Dort, revu à l'aune de la pragmatique) et figure du lecteur (présence d'un lecteur-implicite, même dans un texte de théâtre, en interactions complexes avec le lecteur réel). C'est sur la base de ces rencontres et tensions que peut se développer pleinement l'activité d'actualisation imageante, visuelle et auditive, du sujet lecteur de théâtre, tant dans la fiction que dans la régie, et dans la langue.

Bien sûr, on trouvera dans l'ensemble de ce qui se publie dans des collections jeunesse des œuvres affadissant le regard sur le monde, mais la qualité et la profondeur de ces écritures, dans leur ensemble, frappe l'observateur attentif. J'ai déjà pu montrer, en analysant les thèmes circulant dans près de deux cent cinquante pièces, que rien n'en est exclu des grands sujets qui habitent les préoccupations des adultes, de la vie à la mort, de la guerre à la naissance, des joies familiales au divorce, de la haine à l'amour, de la petitesse humaine aux forces du rêve, de l'enfant porteur de tous les miracles à l'enfant abîmé... Toutes les douleurs et tous les déséquilibres du monde moderne et contemporain, ses pertes de repères, ses guerres insensées occupent une place importante dans le répertoire jeunesse, bien loin de la soumission à un genre comique aseptisé que de nombreux manuels scolaires et certaines éditions ont

Io. P. Szondi, La théorie du drame moderne, Lausanne, L'Âge d'homme, I983.

II. M. Martinez-Thomas (éd.), Jouer les didascalies, Toulouse, Presses universitaires du Mirail, 1999.

I2. Se reporter à la définition synthétique que j'en donne dans le glossaire de Vers un théatre contagieux, ouvr. cité, p. 535 .

I3. M. Couturier, La figure de l'auteur, Paris, Seuil, I995. 
trop cherché à imposer ${ }^{14}$. Le théâtre jeunesse traite même certains sujets que le théâtre généraliste aborde peu, comme les enfants-soldats, le suicide ou la maladie mortelle de l'enfant.

Il suffit de se reporter aux index thématiques de mes deux ouvrages. Les thèmes dominants, pour les II2 pièces analysées dans le premier ${ }^{15}$, sont les suivants : amour (2I pièces), enfance (20 pièces), mort (Is pièces), guerre, rêve (I3 pièces), $\operatorname{conte}^{16}$ (9 pièces), famille, amitié, violence, enfant et imaginaire (8 pièces). Suivent à 5 ou 6 occurrences : divorce, éducation, folie, langage, parole, relations enfant-adulte et vie. Le même travail réalisé dans le volume deux ${ }^{17}$, portant cette fois sur 80 œuvres et io recueils révèle ces prédominances : mort et famille ( 33 pièces), amour et relations adultes/enfants ( 25 pièces), destin, grandir et solitude (20 pièces), politique/société (I9 pièces), violence (I4 pièces), abandon, corps, imaginaire et langue (I3 pièces), différence (I2 pièces), quête (II pièces), conte (Io pièces), apprivoisement, attente et vie (9 pièces). On peut aussi évoquer les occurrences de la maladie d'Alzheimer (6 pièces) à hauteur égale avec la mémoire et la vieillesse.

Les tabous ne sont donc pas dans les sujets abordés, ils sont dans la qualité du mode de détour, excluant la frontalité ou la désespérance. Le seul tabou thématique que je puisse apercevoir est la sexualité, mais elle est cependant présente et c'est plutôt son abord direct qui est forclos.

Sont ainsi clairement posés les liens de l'esthétique et de l'éthique.

L'adresse aux enfants et aux jeunes construit depuis une vingtaine d'années une esthétique théâtrale forte de ses mélanges entre le dramatique et l'épique. Le principe est certes courant dans le théâtre contemporain, mais sa mise en œuvre dramaturgique, au sens premier du mot donné par Joseph Danan ${ }^{18}$, pousse à la créativité du fait de la complexité des jeux de détour à l'œuvre dans le rapport à l'adresse. Telle est en tout cas l'hypothèse que je formule.

14. Se reporter à l'article : «La question des genres dans l'enseignement du théâtre contemporain : courants esthétiques et modèles didactiques, des convergences", dans M. Bernanoce, A. Brillant-Annequin (dir.), Enseigner le théatre contemporain, Grenoble, SCÉRÉN-CRDP de Grenoble, 2009, p. 35-51.

I5. M. Bernanoce, À la découverte de cent et une pièces, ouvr. cité. Il faut préciser que deux thématiques peuvent bien sûr correspondre à la même pièce. Je dois aussi ajouter que les entrées de l'index thématique ont évolué du premier volume au deuxième. Il n'en demeure pas moins que l'ensemble est révélateur des tendances thématiques à l'œuvre.

16. Le conte est ici entendu comme thématique explicite, très souvent abordé dans une forme de mise en abyme.

17. M. Bernanoce, Vers un théâtre contagieux, ouvr. cité.

I8. J. Danan, Qu'est-ce que la dramaturgie?, Arles, Actes Sud, 2010. 


\section{Le rapport au bloc d'enfance}

Avec Enfance obscure ${ }^{19}$, Pierre Péju propose un ouvrage peu commun, constitué à la fois d'évocations personnelles de son enfance, de l'ordre de l'intime, d'analyses des auteurs parlant de leur rapport à l'enfance, de l'ordre du littéraire, et d'analyses de la nature de ce rapport à l'enfance, en particulier en lien avec l'art, de nature philosophique. Il pose ainsi les bases de la notion d'Enfantin, à opposer à l'infantile. L'infantile, c'est le statut social de l'enfant, ce que la société lui donne comme rôle. L'Enfantin, c'est une permanence de l'enfance, bien au-delà de la notion de souvenirs d'enfance que Pierre Péju récuse : l'enfance reste en nous, elle n'est pas un lieu ni un temps autonome que nous pourrions retrouver, faire remonter. C'est ce qu'il appelle l'Enfantôme ou encore le spectre faible $^{20}$, capital dans la démarche même d'écriture, comme il le montre chez Nathalie Sarraute.

La notion d'Enfantin permet d'entériner une vision du théâtre jeunesse comme théâtre modeste : comme nous l'avons vu, il est très difficile pour un auteur d'imaginer les réactions des enfants réels à la lecture de sa pièce, encore moins à la réception de la mise en scène de celle-ci. Le détour que constitue la décentration de l'adulte en quête d'un regard d'enfance constitue une autre sorte d'épique auquel il faut donner toutes ses lettres de noblesse. Le répertoire de théâtre jeunesse constitue ainsi peu à peu, au-delà de lui, un lecteur modèle poussant à l'ouverture et à la générosité littéraires et théâtrales.

L'enfance a ses lois, qui ne sont pas celles des adultes, dans la proximité de la «sauvagerie ${ }^{2 \mathrm{x}}$ » du jeu telle que l'analyse Nietzsche. Loin de l'image du geste de surplomb que le grand public se forge encore trop souvent de l'écriture en direction des jeunes (même si ce geste peut exister), la réflexion de Pierre Péju montre que cette adresse ne fonctionnera vraiment que si l'auteur est lui-même ancré de façon juste dans l'Enfantin.

Mais comment définir cette justesse? La réponse apportée par Pierre Péju tient dans la parabole nietzschéenne des «trois métamorphoses» :

À l'affirmation d'Héraclite selon laquelle "le monde est un enfant qui joue», comme à l'énigme de la Sphinge, fait écho, chez Nietzsche, la fameuse parabole des "trois métamorphoses", dans Ainsi parlait Zarathoustra : "Je vais vous dire trois métamorphoses de l'esprit : comment l'esprit devient chameau, comment le chameau devient lion, et comment enfin le lion devient enfant. Nietzsche fait de

19. P. Péju, Enfance obscure, Paris, Gallimard, 2oII.

20. Ibid., p. I4.

2I. Ibid., p. 313. 
l'enfant le dernier terme de la transformation de l'esprit. On dirait qu'il intervertit à plaisir les différents "stades de la marche» dans l'énigme d'Edipe [...].

La dernière métamorphose, selon Nietzsche, consiste donc à "devenir enfant» autant qu'à devenir artiste ${ }^{22}$.

Aux philosophes qui, dès la fin du ve siècle avant Jésus-Christ, à commencer par Platon, ont considéré que l'enfant était le «monstre» de la philosophie ${ }^{23}$, l'état dont il faut se défaire pour grandir et arriver à maturité et sagesse, Nietzsche oppose, avec Héraclite, ce que Gilles Deleuze analyse ainsi dans Nietzsche et la philosophie:

Affirmer le devenir, affirmer l'être du devenir sont les deux temps d'un jeu qui se composent avec un troisième terme, le joueur, l'artiste et l'enfant. Le joueur-artisteenfant, Zeus enfant. Dionysos, que le mythe nous présente entouré de jeux divins ${ }^{24}$.

L'adresse à des enfants et à des jeunes est alors le lieu d'activation privilégié de l'Enfantin, le terrain de jeux favori du «joueur-artiste-enfant» que peut être l'écrivain s'il a réussi à «devenir enfant».

Le rapport à l'enfance, entendue comme bloc d'enfance, fonctionne ainsi comme un procédé de détour pour dire le monde des plus fictionnalisants, comme un surplus de fictionnalisation au cour de la trajectoire humaine.

\section{Les grandes tendances esthétiques et éthiques du théâtre jeunesse}

\section{Trois grandes voix didascaliques}

Mon travail de synthèse m'a permis de faire émerger trois grands modes de fonctionnement de la théâtralité mêlant épique et dramatique.

Apparaît tout d'abord une voix du conte, au sens de contage, en particulier au travers de trois fonctionnements dramaturgiques particuliers que sont l'adresse didascalique, le récitant fictionnalisé et la ritualisation dramaturgique ${ }^{25}$.

Les fonctionnements de l'adresse didascalique me semblent fondateurs de la voix du conte que l'on pourrait définir comme une forme d'engagement esthétique de l'ordre du dire. Un exemple emblématique en est l'univers de Joël Jouanneau, dans le sillage duquel on peut aujourd'hui placer l'écriture de

\footnotetext{
22. Ibid., p. 3I4-3I5.

23. Ibid., p. 300.

24. Cité par P. Péju, Enfance obscure, ouvr. cité, p. 3I3.

25. Tous les termes utilisés ici en italiques correspondent à des entrées du glossaire des deux ouvrages $\grave{A}$ la découverte de cent et une pièces et Vers un théâtre contagieux, ouvr. cités.
} 
Fabrice Melquiot aussi bien que celles de Jean Cagnard et de Philippe Gauthier et même, en creux par la monstration, celle de Sylvain Levey.

Les procédés de la ritualisation dramaturgique, quant à eux, sont un peu plus subtils à définir mais ils n'en demeurent pas moins facilement discernables, offrant à la voix du conte les moyens rythmiques du balancement, auxquels on peut donner un sens poétique et en quelque sorte archaïque par sa relation au bercement, dans le sillage de la ritournelle deleuzienne. Dans ce domaine, les grandes écritures sont celles de Mike Kenny, de Jon Fosse et de Bruno Castan.

J'ai enfin analysé, en particulier dans Une chenille dans le cœur ${ }^{26}$ de Stéphane Jaubertie et dans La Nuit électrique ${ }^{27}$ de Mike Kenny, l'imbrication complexe entre récitant et personnage que représente ce que j'ai proposé d'appeler le récitant fictionnalisé. J'y discerne une forme de métarécit, de fictionnalisation du geste de dire.

Cette voix du conte, même si elle peut être liée dans certains cas aux contenus thématiques des contes, a son existence propre. Il s'agit bien d'une orientation dans le geste d'écriture théâtrale qui se rapproche de la parole du conteur. Sans doute pourrait-on l'inscrire dans le sillage de la réflexion de Benjamin sur le "raconteur», der Erzähler, par son rapport à l'oralité non reproductible.

J'ai ensuite analysé une voix du relais, définissant une relation de l'auteur au lecteur que l'on pourrait qualifier de reliante, comme un passage de relais, et que l'on pourrait rattacher aux recherches sur l'empathie ${ }^{28}$, dans le sillage des travaux d'Alain Berthoz. La voix du relais est quête et création de liens, prolongeant l'adresse didascalique de façon implicite mais tout aussi forte.

J'ai pu ainsi détecter de nombreux usages intéressants de la nomination des personnages, créant des effets de béance, d'indécision, de surprise, de points de vue, à saveur métathéâtrale. Je me suis ainsi penchée sur Coro $\mathrm{Nero}^{29}$ de Stanislas Cotton, Miche et Drate ${ }^{30}$ de Gérald Chevrolet, Petit ${ }^{31}$ de Catherine Anne, parmi d'autres exemples et j'aurais pu les multiplier tant ils sont nombreux, posant des questions de fond sur l'identification supposée du lecteur/spectateur aux personnages d'enfants (qui par ailleurs ne sont pas une nécessité du genre). Si ces usages de la nomination relèvent d'une voix du relais, c'est qu'ils construisent

26. S. Jaubertie, Une chenille dans le cœur, Montreuil, Éditions Théâtrales Jeunesse, 2008.

27. M. Kenny, La Nuit électrique, Arles, Actes Sud-Papiers, coll. «Heyoka jeunesse», 2008.

28. J. Aden, "L'empathie, socle de la reliance en didactique des langues-cultures», dans J. Aden, T. Grimshaw, H. Penz (dir.), Enseigner les langues-cultures à l'ère de la complexité. Approches interdisciplinaires pour un monde en reliance, Bruxelles, Peter Lang, 2010.

29. S. Cotton, Petites Pièces pour dire le monde 2, Carnières, Lansman, 2008.

30. G. Chevrolet, Miche et Drate, Montreuil, Éditions Théâtrales Jeunesse, 2007.

31. C. Anne, Petit, Paris, L'École des loisirs, coll. "Théâtre», 2002. 
une identification moins centrée sur les personnages eux-mêmes que sur le fait de les nommer, introduisant de ce fait une distance de nature épique. Souvent, la nomination devient même un motif dramaturgique, au sens où le procédé croise la thématique pour mieux faire théâtre. Les écritures jeunesse relèvent très fréquemment d'une performativité mi-épique mi-dramatique.

Cela rejoint les jeux du monodrame, les jeux de rêve et de points de vue que l'on peut remarquer comme notables par exemple dans Bled ${ }^{32}$ de Daniel Danis, dans Kant / Noir et humide / Si lentement / Petite Soeur ${ }^{33}$ de Jon Fosse, dans Louise / les ours ${ }^{34}$ de Karin Serres ou dans Jojo le récidiviste ${ }^{35}$ de Joseph Danan...

Je me suis également penchée sur des exemples intéressants de ce que j'ai appelé le théâtre épistolaire, théâtre par lettres, et du théâtre diariste, théâtre en appui sur un journal intime : Émile et Angèle, correspondance ${ }^{36}$ de Françoise Pillet et Joël Da Silva, L'Enfant cachée dans l'encrier ${ }^{37}$ de Joël Jouanneau ou Les Cahiers de Rémi $i^{3}$ de Dominique Richard.

Émergent ainsi des procédés que je qualifie de relais du récit, très sensibles dans Mange ta main ${ }^{39}$ de Jean-Claude Grumberg pour ce qui est de l'écriture, de façon ironique, et dans Une lune entre deux maisons ${ }^{40}$ de Suzanne Lebeau pour ce qui est de la lecture (la seule pièce jeunesse, à ma connaissance, mettant en jeu l'apprentissage de la lecture).

J'ai enfin distingué une voix du jeu, définissant un ludisme de fond qui en quelque sorte subsume les deux autres voix.

Ce sont les jeux avec l'espace de la page, ce que j'appelle les jeux cotextuels, que cela se manifeste dans les liens texte/image dans les album-théâtre ou dans la simple mise en page du texte.

Ce sont aussi les jeux avec le récit au travers du dénarré et du dédramatisé. J'ai repris la notion de dénarré de Gérald Prince et je l'ai transposée en termes d'analyse dramaturgique : il s'agit donc de ce qui aurait pu se passer dans la fiction mais ne se passe pas tandis que pourtant des indices en sont donnés. J'ai sur ce modèle construit la notion de dédramatisé, cette fois du côté de la régie.

32. D. Danis, Bled, Paris, L'Arche, 2008.

33. J. Fosse, Kant / Noir et humide / Si lentement / Petite Sour, Paris, L’Arche jeunesse, 2009.

34. K. Serres, Louise / les ours, Paris, L'École des loisirs, coll. "Théâtre», 2006.

35. J. Danan, Jojo le récidiviste, Arles, Actes Sud-Papiers, coll. "Heyoka jeunesse», 2007.

36. F. Pillet, J. Da Silva, Émile et Angèle, correspondance, Montreuil, Éditions Théâtrales Jeunesse, 2005.

37. J. Jouanneau, L'Enfant cachée dans l'encrier, Arles, Actes Sud-Papiers, coll. «Heyoka jeunesse», 2009.

38. D. Richard, Les Cahiers de Rémi, Montreuil, Éditions Théâtrales Jeunesse, 2012.

39. J.-C. Grumberg, Mange ta main, Arles, Actes Sud-Papiers, coll. «Heyoka jeunesse», 2006.

40. S. Lebeau, Une lune entre deux maisons, Montréal, Québec/Amérique, 1980; Montreuil, Éditions Théâtrales Jeunesse, 2006. 
Le théâtre de Philippe Dorin tire ainsi la plus grosse partie de son ludisme de ces jeux du dénarré et du dédramatisé. On est sans cesse dedans/dehors dans son théâtre, à l'image de l'introduction presque systématique de chansons en langue étrangère. C'est particulièrement vrai de Abeilles, habillez-moi de vous ${ }^{4 \mathrm{r}}$.

Enfin, me sont apparus les phénomènes particulièrement importants, en termes de pragmatique, de jeu avec le double, qu'il s'agisse du double générationnel ou du double théâtralisét2. Dans le cas du double générationnel, il s'agit de construire l'histoire et la fable autour de deux personnages, l'un enfant ou jeune, l'autre adulte ou âgé, et c'est très fréquent dans l'ensemble du répertoire, non sans clichés dans les pièces les plus "paresseuses". Le double théâtralisé est une variante du précédent en forme de monodrame et d'épique. Le thème du double générationnel se fait motif dramaturgique, le mettant en œuvre dans la structure dramaturgique : un personnage adulte ou âgé se raconte enfant ou adolescent. On peut même avoir trois niveaux temporels ainsi dans Petite Fille dans le noir de Suzanne Lebeau.

Nous allons parcourir maintenant quelques grandes tendances esthétiques plus globales, inscrites dans l'histoire théâtrale et littéraire, qui se dessinent dans l'ensemble du répertoire de théâtre jeunesse.

\section{Des ancrages littéraires et théâtraux}

J'ai tout d'abord distingué une tendance parabolique, dont l'ensemble de l'œuvre théâtrale de Nathalie Papin me semble l'exemple emblématique, de Camino à Un, Deux, Rois, en passant par L'Habitant de l'escalier ${ }^{43}$. À cette tendance générale correspond, en retournant vers les procédés dramaturgiques de détail, la théâtralisation du sens figuré que l'on voit fortement à l'œuvre par exemple dans le recueil de courtes pièces de Suzanne Lebeau Contes d'enfants réels ${ }^{44}$.

Cette propension parabolique, comme "pas de côté» défini par Jean-Pierre Sarrazac dans La parabole ou l'enfance du théâtre ${ }^{45}$, est à opposer à la tendance

4I. P. Dorin, Abeille, habillez-vous de moi, Paris, L'École des loisirs, coll. "Théâtre», 20 oro.

42. Pour de plus amples développements, consulter «Le couple générationnel dans le théâtre jeunesse : une dualité peut en cacher une autre», Recherches et Travaux, $\mathrm{n}^{\circ}$ 86, Jouer (avec) la vieillesse, A. Martinez (dir.), p. 83-92.

43. N. Papin, Camino; Un, Deux, Rois; Petites Formes; L'Habitant de l'escalier, Paris, L'École des loisirs, coll. "Théâtre», 2003; 20I2; 2015.

44. S. Lebeau, Contes d'enfants réels, Montréal, VLB Éditeur, 1995; Montreuil, Éditions Théâtrales Jeunesse, 2009.

45. J.-P. Sarrazac, La parabole ou l'enfance du théâtre, Belval, Circé, 2002. 
didactique que je crois aujourd'hui dépassée mais dont on sent poindre le retour insidieux. Notons que la tendance parabolique fait de l'écriture le creuset d'une recherche de sens, là où la pente didactique assène des vérités. Dire le monde du point de vue de l'enfance, c'est endosser des questionnements métaphysiques.

Dans le prolongement de la voix du relais, émerge ensuite la tendance à la série théâtrale, où l'on peut sans aucun doute retrouver une propriété forte de la littérature de jeunesse. J'y ai tout d'abord distingué les simples suites, telle celle des Bouli Miro ${ }^{46}$ de Fabrice Melquiot, en appui sur un retour des personnages principaux et sur une chronologie, même si dans ce cas précis on a un bel exemple de retour vers les origines avec Bouli Année Zéro. Joseph Danan a fait de même avec sa pièce Le Théâtre des papas ${ }^{47}$. Mais il y a aussi une autre sorte de série, assez unique, avec la totalité des pièces de Dominique Richard qui créent ce que j'ai choisi d'appeler une constellation théâtrale : un personnage mineur dans une pièce devient majeur dans une autre ou l'inverse, et cela construit un réseau qui en quelque sorte s'auto-engendre dans les processus d'écriture de l'auteur, et s'offre à l'imaginaire de son lecteur. On peut retrouver cela, sur un mode atténué, dans des phénomènes d'échos entre deux pièces, qui se mettent à fonctionner en quelque sorte en diptyque : c'est le cas des œuvres de Nathalie Papin Le Pays de Rien ${ }^{48}$ et Un, Deux Rois. On peut discerner dans cette tendance esthétique une portée éthique qui dépasse, me semble-t-il, le simple goût du récit. Il y a là une sorte de transposition dramaturgique des rondes enfantines et du cercle théâtral fondateur, à forte coloration humaniste.

Enfin la troisième grande tendance se construit autour de ce que j'appelle l'engagement multicolore ${ }^{49}$, en m'inspirant de la philosophie tragique de Clément Rosset et de la notion de Joie telle qu'il la définit ${ }^{50}$. J'entends par là une parole forte sur le monde entre rires et larmes, sur des sujets difficiles, dans le refus conjoint de l'abandon à la noirceur et de l'ignorance du tragique. Il s'agit tout à la fois d'éviter le nihilisme à coloration cynique et la distraction pascalienne. Dominique Richard parle ainsi de "tragique joyeux ${ }^{s \mathrm{~s}}{ }$. Wajdi Mouawad de son côté rejette le dramatique au profit du tragique, dont il lui paraît essentiel de faire vivre l'expérience à des jeunes ${ }^{52}$. Si l'on peut parler

\footnotetext{
46. F. Melquiot, Bouli Miro, Paris, L'Arche jeunesse, 2002.

47. J. Danan, Le Théâtre des papas, Arles, Actes Sud-Papiers, coll. «Heyoka jeunesse», 2015.

48. N. Papin, Le Pays de Rien, L'École des loisirs, coll. "Théâtre", 2002.

49. "Le répertoire de théâtre jeunesse entre humour noir et humour multicolore», Humoresques, $\mathrm{n}^{\circ} 30$, L'enfance du rire, N. Feuerhahn (dir.), 2009, p. II9-133.

50. C. Rosset, La philosophie tragique, Paris, PUF, I960; La force majeure, Paris, Minuit, 1983.

5I. M. Bernanoce, Vers un théâtre contagieux, ouvr. cité, p. 44I.

52. "Écrire par bouffées d'enfance, un tragique joyeux", dans M. Bernanoce, Vers un théattre contagieux, ouvr. cité, p. 44I.
} 
d'engagement, c'est que les auteurs prennent à bras-le-corps des sujets moraux sans faire la morale. C'est le cas des enfants-soldats avec Le bruit des os qui craquent de Suzanne Lebeau. C'est le cas du suicide de l'enfant ou du jeune, avec Du temps que les arbres parlaient ${ }^{53}$ d'Yves Lebeau ou Debout ${ }^{54}$ de Nathalie Papin. Dans la même veine, mais dans une forme plus nette encore de dramede-la-viess, Petit Pierre ${ }^{56}$ de Suzanne Lebeau confronte la vie d'un homme handicapé qui se met à créer un manège tournant rond tandis qu'autour de lui, tout au long de sa vie, c'est un manège de mort et d'injustice qui ne tourne pas rond et démolit bien des vies. Dans une approche assez comparable, Costa le Rouge de Sylvain Levey entrelace trois générations et confronte la petite misère humaine aux grandes forces telluriques et épiques de l'histoire.

L'exploration du répertoire de théâtre jeunesse a pu ainsi montrer combien sa vitalité est grande, fortement empreinte d'une joie du récit, de l'adresse, $\mathrm{du}$ fait de dire et de nommer qui le connecte à son lecteur. Les indices de ce relais constituent autant de portes d'entrée par lesquelles le lecteur est clairement invité à venir prendre place dans cet enchaînement de points de vue et de paroles prises, données et reçues. Peut-être n'est-il finalement question que de jouer à la vie, d'en mimer les jeux, qu'ils soient de pages et de mots, de raconté, de tu et de presque raconté, entre naissance et vieillesse. L'ombre portée de cet univers touche à la mort.

La joie nietzschéenne qui refuse «l'enfant-monstre» de la philosophie relève sans doute d'un ludisme primordial qui met en jeu le "joueur-artiste-enfant» présent dans toute parole littéraire et théâtrale. Ce ludisme colore la dimension métathéâtrale qui en découle d'une saveur toute particulière, habitée, loin des effets formalistes des années 1970.

Ce seul constat a valeur éthique et esthétique et il rend le théâtre jeunesse nécessaire à la vie présente de la littérature dramatique et du théâtre, comme il rend le théâtre nécessaire à la vie présente de la littérature. Les inventivités esthétiques de ce théâtre s'inscrivent dans un rapport à la transmission qui me semble pouvoir contribuer à l'avenir du théâtre.

53. Y. Lebeau, Du temps que les arbres parlaient, Montreuil, Éditions Théâtrales Jeunesse, 2009.

54. N. Papin, Debout, Paris, L'École des loisirs, coll. "Théâtre», $200 \mathrm{I}$.

55. J.-P. Sarrazac, Poétique du drame moderne, Paris, Seuil, 2012.

56. S. Lebeau, Petit Pierre, Montréal, Lanctôt Éditeur, 2002; Montreuil, Éditions Théâtrales Jeunesse, 2006. 


\section{L'effet Petit Poucet : dire le monde aujourd'hui}

Apparaît aussi, depuis quelques années, une tendance que j'ai appelée l'effet Petit Poucet ${ }^{5}$. On est en effet frappé par la forte résurgence de la figure du Petit Poucet. Nombreuses sont les pièces qui réécrivent le conte; nombreuses sont celles qui, plus indirectement, se donnent pour héros un enfant abandonné et qui évoquent les cailloux blancs du conte. Un sociologue pourrait analyser cette tendance. Pour ma part, j'y vois non pas une nouveauté mais l'accentuation d'une tendance moderne et contemporaine des adultes à se sentir perdus, sans transcendance, sur les chemins de leur vie marqués par la peur d'un ogre dévoreur, dont l'engagement de bien des pièces jeunesse dessine la figure sociale et économique.

Dans son ouvrage Petite Poucette ${ }^{5}$, Michel Serres, avec un grand optimisme, discerne dans la troisième grande révolution que nous sommes en train de vivre avec l'invention de l'ordinateur, après celles de l'écriture et de l'imprimerie, les conditions d'une nécessité à se dépasser. Il use pour cela d'une parabole, celle de saint Denis dont on vient de couper la tête et qui la prend sous son bras pour continuer sa route. De la même façon, l'homme contemporain n'a pas d'autre choix que de poursuivre sa route alors que sa tête, qui a été coupée, est tombée dans son ordinateur. Les Petits Poucets et Petites Poucettes d'aujourd'hui semblent ainsi partis pour un futur dont ils ne pourront que se montrer dignes.

Il n'est pas certain que l'ensemble du théâtre jeunesse porte et donne cette vision du monde futur, mais il n'en demeure pas moins que le fonde un tel rapport à une forme d'humanisme. Si la noirceur est très présente dans le répertoire de théâtre jeunesse, elle est ancrée dans un cheminement de vie obstiné, à l'image du Petit Poucet. Le succès public de la réflexion d'Alexandre Jollien, ainsi dans son Petit traité de l'abandon ${ }^{59}$, montre combien l'alliance d'une sensation d'abandon et d'un cheminement de vie impérieuse semble répondre à un actuel besoin de sens. Dans le rapport à l'Enfantin, tel que défini par Pierre Péju, le théâtre jeunesse me semble répondre à ce besoin :

Accueillir l'Enfantin, c'est toujours tenter d'empêcher, désespérément peut-être, le grand massacre du passé.

57. Voir ainsi mon article «Place et nature des adaptations de contes dans le répertoire de théâtre pour la jeunesse : questions posées aux esthétiques théâtrales contemporaines ", Revue d'Histoire du Théâtre, $\mathrm{n}^{\circ} \mathrm{I}-2$, Le conte à l'épreuve de la scène contemporaine, XX-XXI' siècles", 20I2, p. I7I-I8O.

58. M. Serres, Petite Poucette, Paris, Le Pommier, 2012.

59. A. Jollien, Petit traité de l'abandon, Paris, Seuil, 2012. 
Écrire l'Enfantin relève d'une façon de vivre fondée sur le rappel des possibles ${ }^{60}$. C'est pourquoi l'Enfantin ne s'accueille pas dans la passivité. Il invite à passer à l'acte. Quel acte? Chacun le sien, bien sûr. Une occasion offerte à notre maturité de démultiplier les sensations et d'élargir les significations ${ }^{61}$.

Le théâtre jeunesse relève ainsi d'une éthique des possibles qui appelle à l'acte.

À la faveur de l'étude des motifs dramaturgiques que j'ai définis, alliant thématiques et procédés, et dont j'ai commencé à cerner les contours, m'est apparu un motif, la métamorphose, dont j'ai de plus en plus tendance à percevoir la valeur fondatrice. Il me semble en effet que s’y loge la capacité de ce théâtre, qui est sa nécessité, à lutter contre le «massacre du passé», personnel et collectif. Se dessine ainsi une éthique des possibles qui est aussi une esthétique. Le motif dramaturgique de la métamorphose, qui semble subsumer les autres, dit en le faisant que le possible est toujours là, à portée de regard dans la lecture, à portée de mains dans l'écriture.

Mais il ne faudrait pas se tromper ni se leurrer sur la nature de l'acte accompli par le rapport à l'Enfantin. Suzanne Lebeau en témoigne avec lucidité :

Écrire pour les enfants est davantage une façon de regarder le monde que la nécessité d'en inventer un, différent et merveilleux, plus gai, plus coloré, plus souriant. [...] Quand je pense que j'écris pour les enfants, je ne crois pas recréer le monde; j'adopte seulement un point de vue ${ }^{62}$.

C'est avant tout de la métamorphose du récit qu'il est question, et non du monde. Bien sûr, et le fort engagement de bien des écritures jeunesse en témoigne, les écrivains de théâtre jeunesse croient un peu, malgré tout, que le théâtre peut apporter sa pierre à la construction du monde. Mais sans doute la figure de l'auteur de théâtre jeunesse construit-elle avant tout, c'est le sens de la forte adresse, une relation privilégiée aux enfants en tant que sujets uniques. C'est ce qu'exprime Philippe Dorin :

J'écris pour les enfants mais peut-être qu'il est tout seul. J'écris pour un seul enfant assis au milieu de cent quatre-vingt-dix-neuf autres, dans la salle du théâtre, et il se dit : «ça y est, cette fois, c'est pour moi»"

Les jeux d'adresse représentent une invitation à endosser un point de vue, pour s'enrichir de tous ses possibles et en ouvrir d'autant son rapport au monde et à la langue, et cela est vrai aussi de l'auteur adulte et du lecteur adulte. Du

6o. P. Péju, Enfance obscure, ouvr. cité, p. 47.

6I. Ibid., p. 57.

62. S. Lebeau, dans F. Villaume (dir.), Le choix de Suzanne Lebeau, Montreuil, Éditions Théâtrales, 2013, p. 79.

63. M. Bernanoce, À la découverte de cent et une pièces, ouvr. cité, p. I49. 
coup, cette figure de l'auteur appelle une figure de la scène à son image. Là est aussi sa force de contagion.

\section{Ouvrir des chemins}

Le théâtre jeunesse semble aujourd'hui la partie consolante privilégiée du théâtre contemporain. Le réalisme mineur à l'œuvre dans ce répertoire, dans son fort rapport au récit, à la transmission et au ludisme, ouvre la voie à un renouvellement du théâtre, tout du moins y contribue-t-il. Sans l'affirmer, il paraît cependant légitime d'en avancer l'hypothèse.

Les dramaturgies jeunesse, que l'on peut qualifier de dramaturgies de la contagion tant elles construisent des ponts entre auteur et lecteur, s'offrent ainsi, à qui sait les prendre comme elles nous font, avec tout l'humanisme poétique auquel conduit l'Enfantin.

Il apparaît qu’à la question soulevée par Adorno en 1955 dans Prismes, "écrire un poème après Auschwitz est barbare ${ }^{64}$ ", le répertoire de théâtre jeunesse répond par la nécessité, au sens philosophique du terme, d'un ancrage dynamique dans le futur. Benjamin dans Sur le concept d'histoire remettait en cause une conception linéaire du temps historique, tournée vers le futur, car il lui semblait qu'ainsi on rate l'à-présent, qui est rendez-vous avec le passé. Il voyait dans l' «Angelus novus» de Klee un "Ange de l'histoire» avançant vers le futur à reculons, horrifié par les décombres de la catastrophe $e^{65}$. Les écritures théâtrales jeunesse, par le jeu complexe de points de vue intériorisé du fait de la relation à l'Enfantin, réussissent à être à ce point de rendez-vous, comme l'ange du dieu bonheur de Brecht, par une forme de regard oblique, détaché de la contemplation et de la fascination un rien morbides que peut générer le regard frontal porté sur les catastrophes.

Clément Rosset dans La force majeure discerne avec Nietzsche deux sortes de ruminants :

Ceux qui ruminent sans cesse mais sans réussir à digérer (cas de l'homme du ressentiment), et ceux qui ruminent et digèrent (cas de l'homme dionysiaque). Mauvais et bons ruminants. [...] Mais ce n'est pas là exactement ce que pense Nietzsche en matière de rumination. À y regarder de plus près, la répartition des rôles est assez différente : le bon ruminant a accès tout à la fois au bonheur et au

64. T. W. Adorno Prismes [Prismen, 1955], Paris, Payot, 1986.

65. W. Benjamin, Sur le concept d'histoire IX. Euvres III, 1940, trad. M. de Gandillac, Paris, Gallimard, coll. «Folio Essais», I97I, p. 434. 
malheur, et le sort du mauvais ruminant est de n'avoir accès ni à l'un ni à l'autre. Car il ignore le bonheur puisqu'il n'arrive pas à digérer le malheur ${ }^{66}$.

Les auteurs de théâtre jeunesse seront donc heureux d'apprendre qu'ils sont majoritairement de «bons ruminants» nietzschéens : leurs écritures s'inscrivent dans un geste de transmission du chemin en forme de contagion joyeuse.

66. C. Rosset, La force majeure, ouvr. cité, p. $4 \mathrm{I}$. 